Nouveaux cahiers de la recherche en éducation

\title{
La problématisation des apprentissages en enseignement de l'histoire-géographie : quels cadres de référence, quels fondements?
}

\section{Johanne Lebrun et Daniel Niclot}

Volume 15, numéro 1, 2012

La problématisation des apprentissages en enseignement de l'histoire-géographie : quels cadres de référence, quels fondements?

URI : https://id.erudit.org/iderudit/1013375ar

DOI : https://doi.org/10.7202/1013375ar

Aller au sommaire du numéro

Éditeur(s)

Faculté d'éducation, Université de Sherbrooke

ISSN

1911-8805 (numérique)

Découvrir la revue

Citer ce document

Lebrun, J. \& Niclot, D. (2012). La problématisation des apprentissages en enseignement de l'histoire-géographie : quels cadres de référence, quels fondements? Nouveaux cahiers de la recherche en éducation, 15(1), 3-4. https://doi.org/10.7202/1013375ar d'utilisation que vous pouvez consulter en ligne. 


\title{
La problématisation des apprentissages en enseignement de l'histoire-géographie: quels cadres de référence, quels fondements?
}

\author{
Johanne Lebrun \\ CRCIE-CREAS, Université de Sherbrooke \\ et Daniel Niclot \\ Université de Reims
}

La question de la problématisation en histoire et géographie dans l'enseignement primaire et secondaire est abordée selon deux grandes entrées. La première est celle de l'observation et de l'analyse de situations de classe ou de formation professionnelle. La seconde est celle d'études extérieures aux situations de classe qui portent sur l'analyse de manuels scolaires ou sur une enquête réalisée auprès d'enseignants. Malgré la diversité des contextes nationaux, des niveaux d'enseignement étudiés, des méthodologies et des grilles de lectures mobilisées, les auteurs soulignent l'intérêt de la problématisation, notamment pour donner du sens aux savoirs scolaires et pour favoriser l'apprentissage des élèves. Ils insistent aussi sur le défi que constitue la mise en œuvre de démarches problématisées qui se heurte à de nombreux obstacles, analysés et identifiés dans la plupart des articles.

L'article de Jean-François Thémines et les deux articles suivants étudient la problématisation en analysant des situations d'enseignement-apprentissage. Les deux situations présentées dans ce premier article concernent la formation de professeurs du secondaire. Les séances analysées sont organisées autour de questionnements favorisant la réflexion sur l'unité et sur la différenciation de l'espace, de nature à relever, selon l'auteur, le défi de la problématisation. En référence à l'épistémologie de la discipline scientifique, le rôle de la problématisation dans une géographie scolaire française centrée sur les acteurs est d'abord présentée. L'auteur définit ensuite la finalité de cette discipline qui est de donner aux élèves des outils pour qu'ils puissent, comme citoyens, agir sur l'espace public. Remettant en cause les différents modèles qui décrivent la manière dont se construisent les savoirs scolaires, il propose celui de la composition didactique qui prend en compte la multiplicité des savoirs mobilisés dans les leçons de géographie.

La problématisation des leçons de géographie est préconisée par les programmes à l'école primaire en France depuis quelques années, note Thierry Philippot. Il s'interroge à la fois sur la définition de la problématisation et sur les objectifs de cette démarche qui, selon lui, participe à la construction par les élèves du sens des savoirs scolaires. La recherche s'appuie sur des 
enregistrements vidéoscopiques de séances de géographie et des entretiens d'autoconfrontation simples. Les résultats mettent en évidence les difficultés qu'ont les maîtres polyvalents du primaire à intégrer la démarche de problématisation en géographie.

Roselyne Lebourgeois et Catherine Rebiffé étudient, quant à elles, la problématisation en classe d'histoire à l'école primaire, en centrant leurs analyses sur les dialogues entre élèves et sur les mouvements discursifs qu'ils suscitent. Pour les autrices, l'une des façons de prendre du recul avec l'énoncé historique est de s'interroger sur la langue et le vocabulaire utilisés. Dans le cadre d'une recherche collaborative associant des didacticiens de français et d'histoire et l'enseignante de la classe, les données prélevées sont constituées par les transcriptions de deux situations d'enseignement-apprentissage organisées à partir de problèmes construits pour dépasser les préjugés des élèves. Cette étude est l'occasion de réfléchir sur les liens entre parole et pensée, sur la dynamique des argumentations et sur les tensions qui naissent de l'opposition entre les différentes cultures personnelles (scolaire, familiale...) que le maître doit gérer.

Les deux articles suivants n'analysent pas les pratiques de classes, mais s'appuient sur l'analyse de manuels et sur les représentations des enseignants pour le dernier texte.

L'épistémologie de la science géographique et des savoirs scolaires, la didactique et les évolutions de la réflexion pédagogique entrent en convergence durant les années 1980 en France pour faire de la problématisation la pièce maîtresse de la rénovation de la géographie scolaire. Daniel Niclot analyse la problématisation dans les manuels scolaires récents de l'enseignement secondaire à l'aune de ces évolutions. Il montre que conformément aux recommandations des programmes, des problématiques explicites figurent dans les manuels en début de chapitre. Pourtant, même si des problématiques existent dans les manuels analysés, elles ne participent pas au développement d'une réflexion autonome sur l'espace par les élèves et elles masquent en fait une logique d'exposition qui demeure largement transmissive.

Mathieu Bouhon, enfin, aborde la question de la problématisation par une enquête menée auprès de 175 enseignants d'histoire belges et luxembourgeois sur leurs préparations de leçons. Il met en relation leurs choix pédagogico-didactiques et les modèles auxquels ils adhèrent avec leurs représentations de la discipline qu'ils enseignent. Tout en reconnaissant l'intérêt de la problématisation, la plupart d'entre eux ne mettent pas la découverte et le questionnement par les élèves au centre de leurs préoccupations. Cet article soulève une question qui est implicitement au cœur de la plupart des articles de ce numéro, celle de la prégnance des représentations sociales des enseignants sur leurs choix pédagogico-didactiques, et au-delà, celle de l'adéquation de leur formation professionnelle. 